\title{
RELAÇÃO MÉDICO-PACIENTE DURANTE A ENTREVISTA CLÍNICA: PERCEPÇÃO DO SURDO
}

\author{
MEDICAL-PATIENT RELATIONSHIP DURING CLINICAL INTERVIEW: PERCEPTION OF DEAF
}

\begin{abstract}
MARQUES, Yndri Frota Farias ${ }^{1 *}$; MEDEIROS, Carolina Lustosa De ${ }^{1}$; ARANTES, Samuel Borges ${ }^{1}$; FONTENELE, Khalina Assunção Bezerra ${ }^{1}$; NASCIMENTO, Clesivane Do Socorro Silva Do ${ }^{1}$; VASCONCELOS, Any Carolina Cardoso Guimarães ${ }^{1}$
\end{abstract}

1 Instituto de Educação Superior do Vale do Parnaíba - IESVAP, Parnaíba, Brasil.

\begin{abstract}
RESUMO
Introdução: A formação médica anterior às Diretrizes Curriculares de 2014 não se constituía de um olhar integral e humanístico na relação médico e paciente com surdez, pois a aprendizagem de LIBRAS não estava inserida na grade curricular, contribuindo para um maior despreparo e distanciamento dos médicos com a pessoa surda. Sendo assim, o objetivo deste trabalho foi identificar a percepção do paciente surdo acerca da relação médicopaciente estabelecida no atendimento e cuidado médico durante a anamnese clínica. Métodos: Trata-se de um estudo quanti-qualitativo de caráter analítico descritivo. Foram entrevistadas pessoas surdas maiores de 18 anos, de ambos os sexos, com diagnóstico de surdez severa e que fazem uso da Língua de Sinais para se comunicar. As entrevistas foram gravadas em vídeo e posteriormente analisadas a partir da análise de conteúdo proposta por Bardin e transcritas em língua portuguesa pelos pesquisadores. O número de sujeitos foi definido a partir do ponto de saturação das informações obtidas com as entrevistas. Resultados: Oito surdos foram entrevistados, sendo dois homens e seis mulheres. Os surdos responderam doze questões referentes as suas percepções e sentimentos. A partir das respostas e análise temática foram identificadas as seguintes categorias: 1) Sentimentos frente à barreira comunicacional; 2) Comunicação médico - paciente surdo durante a entrevista clínica e exame físico; 3) Inexistência da abordagem centrada na pessoa; 4) Presença ou ausência de acompanhante na consulta; 5) Sentimentos e expectativas frente a um médico fluente em LIBRAS; 6) Meios de comunicação utilizados pelos médicos durante a consulta. Os sentimentos prevalentes durante o atendimento foram tristeza, medo e insegurança. Conclusão: A relação médico-paciente é proveitosa quando existe uma comunicação em que ambas as partes se compreendem, e isso, pode ser efetivado pelo domínio de LIBRAS pelo profissional, contribuindo para uma assistência humanizada, centrada no paciente e na experiência da doença.
\end{abstract}

Palavras-chave: Atenção à Saúde. Barreiras Comunicacionais. Língua de Sinais. Relação MédicoPaciente. Surdez.

\begin{abstract}
Medical training prior to the 2014 Curriculum Guidelines did not constitute an integral and humanistic view of the relationship between doctor and patient with deafness, as learning LIBRAS was not included in the curriculum, contributing to a greater unpreparedness and distance from doctors with the deaf person. Therefore, the objective of this study was to identify the perception of the deaf patient about the doctor-patient relationship established in medical care and care during clinical anamnesis. This is a quantitative and qualitative study with a descriptive analytical character. Deaf people over 18 years of age, of both sexes, with a diagnosis of severe deafness and who use Sign Language to communicate were interviewed. The interviews were videotaped and subsequently analyzed based on the content analysis proposed by Bardin and transcribed in Portuguese by the researchers. The number of subjects was defined from the point of saturation of the information obtained from the interviews. Eight deaf people were interviewed, two men and six women. The deaf answered twelve questions regarding their perceptions and feelings. From the responses and thematic analysis, the following categories were identified: 1) Feelings facing the communication barrier; 2) Medical communication - deaf patient during the clinical interview and physical examination; 3) No person-centered approach; 4) Presence or absence of a companion at the consultation; 5) Feelings and expectations towards a doctor fluent in LIBRAS; 6) Means of communication used by doctors during the consultation. The prevalent feelings during care were sadness, fear and insecurity. The doctor-patient relationship is beneficial when there is a communication in which both parties understand each other, and this can be accomplished by the LIBRAS domain by the professional, contributing to a humanized care, centered on the patient and the experience of the disease.
\end{abstract}

Keywords: Health Care. Communication Barriers. Sign language. Doctor-Patient Relationship. Deafness 


\section{INTRODUÇÃO}

A comunicação é a essência de um exame clínico eficaz, pois através dela, o médico detecta não somente sintomas, mas também os sinais do paciente, uma vez que estes constituem-se de aspectos subjetivos e só podem ser identificados através de uma relação médico-paciente com qualidade e assistência adequada por meio da interação comunicacional ${ }^{1}$. Somente dessa forma, a mensagem do paciente surdo será compreendida sem que haja comprometimento do diagnóstico e da ação terapêutica.

Compreender a importância da comunicação verbal e não verbal é de suma relevância para todos os profissionais, pois é a partir disso que se consegue alcançar as relações interpessoais, acarretando no compartilhamento de informações entre o emissor e 0 receptor ${ }^{2}$. Além disso, no entendimento desse compartilhamento, alcança-se experiência, sendo esta, a base essencial para adquirir o conhecimento. Percebe-se assim, que o compartilhamento de informações se faz necessário em qualquer tipo de interação, especialmente quando se diz respeito a relação médico-paciente, visando um atendimento holístico e humanizado, bem como diálogo eficaz com o usuário do serviço de saúde para uma medicina mais resolutiva. Dessa forma, uma pessoa diagnosticada com surdez, necessita deste tipo de atendimento principalmente no que diz respeito a equidade e integralidade ${ }^{3}$.

A LIBRAS (Língua Brasileira de Sinais), como uma forma de comunicação e expressão que utiliza o sistema linguístico de natureza visual-motora com a sua própria gramática, é reconhecida como meio legal de comunicação, representando um marco na luta da comunidade surda e a garantia de inclusão social 4,5. A lei rege que as instituições públicas e empresas concessionárias de serviços públicos de assistência à saúde devem garantir atendimento e tratamento adequado às pessoas com surdez de acordo com as normas legais ${ }^{6}$. Apesar disso, observa-se no cenário atual, a escassez do conhecimento da Língua de Sinais pelos profissionais médicos e a falta de intérpretes dentro de Unidades Básicas e Hospitais, o que deveria ser garantido por lei e que gera, consequentemente, desafios de acessibilidade, especialmente no que tange os princípios doutrinários e organizativos do SUS (Sistema Único de Saúde), defendidos pela Constituição Federal de 1988 e regulamentados pela lei $n^{\circ} 8.080 / 1990^{6}$. O paciente surdo, deve ter a garantia do direito à ética médica relacionada ao sigilo médico $e$, as informações fornecidas ao profissional de saúde devem ser mantidas em prontuário, revestindo-se em caráter de confidência.
O paciente pode transmitir todas as suas intimidades, sem constrangimento ou medo de ter exposição para o meio social ${ }^{7}$. Dados do IBGE (Instituto Brasileiro de Geografia e Estatística) de 2010, contabilizaram 9,7 milhões de pessoas com características de perda auditiva, sendo um fato preocupante, visto que a maioria desses indivíduos, cerca de um milhão, são jovens com menos de 19 anos de idade ${ }^{8}$. Ou seja, há um número alarmante de pessoas com surdez que buscam os serviços de saúde e que para comunicar-se utilizam a LIBRAS, sendo esta língua pouco ou não utilizada por médicos. Dessa forma, a falha na comunicação pode gerar graves problemas, colocando em risco a vida do paciente, isso porque o surdo tem dificuldade sobre o uso correto do medicamento e sua posologia e os médicos através de uma anamnese pobre em sintomas subjetivos podem provocar erros no diagnóstico ${ }^{9}$.

A formação médica anterior às Diretrizes Curriculares de 2014 não se constituía de um olhar integral e humanístico na relação médico e paciente com surdez, não havendo a disciplina de LIBRAS como eletiva ou obrigatória na grade curricular do acadêmico, contribuindo para um maior despreparo e distanciamento dos médicos com a pessoa surda ${ }^{10}$.

Em virtude disso, é pertinente que esses profissionais adquiram proficiência na Língua de Sinais, como forma de integração dos surdos não somente no âmbito da saúde, mas também da educação e da cultura. Faz-se necessário também a análise e a construção discursiva do vínculo estabelecido entre médico e o paciente surdo, além de investigar como essa relação acontece, para viabilizar um atendimento humanizado ${ }^{9}$.

O objetivo deste trabalho foi identificar a percepção do paciente surdo acerca da relação médico-paciente estabelecida no atendimento e no cuidado médico durante a anamnese clínica.

\section{MÉTODOS}

Trata-se de um estudo quanti-qualitativo, de natureza aplicada com caráter de pesquisa exploratória, uma vez que utilizou o conhecimento adquirido para aplicação na sociedade ${ }^{11}$. O método de análise empregado nessa pesquisa foi o de Bardin, devido ao uso da análise da comunicação através de técnicas que permitem a inferência de conhecimentos relativos existentes na interação entre os indivíduos. Nessa situação, foi utilizado fechamento por saturação teórica, onde aconteceu a suspensão de inclusão de novos participantes quando os dados obtidos começaram a apresentar redundância ${ }^{12}$. 
No que se refere à coleta de informações, esta foi realizada através de entrevista com a comunidade surda da cidade de Parnaíba, Piauí. O grupo de pesquisados foi constituído por pacientes maiores de 18 anos, do sexo masculino e feminino, com diagnóstico de surdez severa comprovado por laudo médico e que fazem uso da LIBRAS.

Incialmente os pesquisadores foram a campo implementar as ações previstas no projeto referentes à coleta de dados através da busca ativa de pacientes surdos em suas residências e no IESVAP (Instituto de Educação Superior do Vale do Parnaíba) na cidade de Parnaíba. Posteriormente, em reunião, as pessoas com surdez foram convidadas a participar da pesquisa, bem como foram esclarecidas em relação aos objetivos da mesma, e, manifestando seu aceite, assinaram o Termo de Consentimento Livre e Esclarecido, que foi aprovado pelo Comitê de Ética em Pesquisa pela Plataforma Brasil com 0 CAAE 96423118.3.0000.0014. Nesse contexto, foram marcadas data e hora para a entrevista, de acordo com a disponibilidade dos sujeitos. Os dados foram coletados pelos pesquisadores através de entrevista utilizando a LIBRAS para subsequentes respostas em questionário sociodemográfico e em roteiro de entrevista semiestruturado. Não havia intérprete no momento da entrevista, porém estavam presentes pessoas de apoio afetivo dos surdos e que conheciam a LIBRAS (amigo ou familiar).

Seguindo a isso, as respostas foram transcritas em português para o Miscrosoft Word 2010 respeitando a construção sintática dos entrevistados e analisados segundo análise temática do conteúdo ${ }^{13}$. O procedimento de busca de informações, baseou-se nas diretrizes e normas regulamentadoras de pesquisas envolvendo seres humanos, número 466, de 2012.

Por fim, os pesquisadores preencheram o roteiro e as descrições de atendimento foram registradas por meio de vídeos, em que os surdos assinaram o Termo de Autorização de Uso de Imagem e Voz. Mais tarde, todas as informações coletadas foram mantidas sob a guarda da orientadora responsável pelo projeto. Ademais, a identidade dos entrevistados foi mantida sob sigilo e confidencialidade.

\section{RESULTADOS E DISCUSSÃO}

A pesquisa teve um grupo de trabalho de oito entrevistados, sendo dois homens e seis mulheres, com idade entre 29 e 41 anos, procedentes da cidade de Parnaíba, Piauí, possuindo diagnóstico de surdez comprovada, sendo um paciente portador da forma adquirida. Dos entrevistados, cinco eram solteiros, dois casados e um divorciado. Aliado a isso, apenas três entrevistados trabalham e somente um possui ensino superior completo, sendo este o paciente com surdez adquirida, os outros cinco entrevistados estão desempregados, nos quais quatro têm ensino fundamental incompleto, dois, ensino médio incompleto e um, ensino superior incompleto. Ademais, cinco pacientes recebem aposentaria por invalidez, o que os "impede" de trabalhar e nenhum deles reconhece a UBS como porta de entrada na rede de atenção. Esse desconhecimento, ocorre devido à redução de busca ativa por Agentes Comunitários de Saúde (ACS), bem como da equipe multidisciplinar aos pacientes vulneráveis, implicando na procura reduzida das pessoas com surdez ao atendimento médico.

Foram feitas um total de 12 perguntas baseadas em questionário semiestruturado a respeito da percepção do paciente com surdez sobre a assistência médica. Com isso, foram categorizadas nos seguintes temas: 1) Sentimentos frente à barreira comunicacional; 2) Comunicação médico - paciente surdo durante a entrevista clínica e exame físico; 3) Inexistência da abordagem centrada na pessoa; 4) Presença ou ausência de acompanhante na consulta; 5) Sentimentos e expectativas frente a um médico fluente em LIBRAS; 6) Meios de comunicação utilizados pelos médicos durante a consulta.

\section{CATEGORIA 1: SENTIMENTOS FRENTE À BARREIRA COMUNICACIONAL}

A falta do conhecimento da LIBRAS pelos médicos dificultou a comunicação com os surdos. Os entrevistados relataram alguns sentimentos durante a entrevista clínica, sendo prevalentes a tristeza, o medo e a insegurança, evidenciado nas falas a seguir.

Entrevistado 1: "Se estou me sentindo mal por uma doença, me sinto ainda pior durante o atendimento por não ser compreendido."

Entrevistado 4: "Me sinto triste, pois o médico não olha para mim do início ao fim da consulta. Ele apenas fala com minha mãe e eu não entendo absolutamente nada. "

Entrevistado 7 "Eu sinto tristeza, não por estar doente, mas porque o médico não olha para mim e então me sinto mal por ser surda."

Entrevistado 8: "Me sinto triste e insegura porque o médico não fala comigo. "

CATEGORIA 2: COMUNICAÇÃO MÉDICO - PACIENTE SURDO DURANTE A ENTREVISTA CLÍNICA E EXAME FÍSICO 
Dos oito entrevistados, a maioria relatou que a relação com 0 médico era distante, pois este não entendia 0 que o paciente surdo estava sentindo. Além disso, foram evidenciados sentimentos de desprezo e indignação por parte dos surdos pelo fato dos médicos conversarem e olharem diretamente para o acompanhante, excluindo o paciente da consulta.

Entrevistado 1: "A relação com o médico foi ruim desde o início. A comunicação estava difícil e não tinha como ele ser meu amigo. Eu gosto apenas quando vai um intérprete comigo".

Entrevistado 2: "Espere, eu sou surda, pare por favor!" (Ele continuava falando e em seguida, fez o exame físico e algumas anotações). "

Entrevistado 3: "O médico fala com minha mãe, não comigo. Ele fala com ela e eu fico esperando, observando, sem entender nada. Quando vou ao médico ele faz o exame físico às vezes, mas não me explica sobre."

Entrevistado 4: "No passado, eu considerava os médicos como amigos, acho que isso acontecia porque nos entendíamos, mas agora os médicos falam e eu não entendo. Então, acho isso ruim, não tenho como criar vínculo com alguém que não me entende (face de raiva). "

Entrevistado 5: "O médico não falou quando foi fazer o exame, quando chegou na hora, ele só colocou um aparelho em mim, sem explicar nada sobre isso."

Entrevistado 6: "Meu marido tem vínculo com o médico, eu não. Eu me sinto desprezada pelos médicos ouvintes."

Entrevistado 7: "Eu realmente me sinto ignorada. Preciso de um intérprete em todas as consultas, não sei quando isso vai mudar, por isso não sou amiga dos médicos."

\section{CATEGORIA 3: INEXISTÊNCIA DA ABORDAGEM CENTRADA NA PESSOA}

Durante a consulta, foi referido por todos os surdos que os médicos não questionavam sobre os vínculos $e$ as relações familiares, despertando nos pacientes sentimentos negativos.

Entrevistado 1: "O médico não perguntou sobre minha família (face de tristeza). Eu e minha mãe brigamos muito, tem muita briga em casa, mas o médico não quis saber disso."
Entrevistado 4: "Nunca nenhum médico me perguntou se eu passava por problemas familiares ou problemas pessoais."

Entrevistado 7: "Na minha família tenho vários problemas porque eu sou a única surda, me sinto incompreendida, mas o médico nunca perguntou sobre isso."

Entrevistado 8: "A comunicação com o médico é ruim e ele não pergunta nada de família, pergunta apenas o que eu sinto para minha amiga intérprete e depois me manda embora."

\section{CATEGORIA 4: PRESENÇA OU AUSÊNCIA DE ACOMPANHANTE NA CONSULTA}

A presença no momento da consulta de um acompanhante, teve aspecto positivo, pois os surdos não se sentiam envergonhados e ficavam mais seguros devido ao acompanhante servir como um facilitador da comunicação entre o médico e o entrevistado.

Entrevistado 1: "Não costumo ir sozinho, mas sim com um amigo ou com minha mãe, eles que falam com o médico por mim. Não tenho vergonha deles, pois me ajudam."

Entrevistado 2: "Quando vou sozinha, cumprimento o médico e explico o que estou sentindo, mas ele não entende nada. Digo que estou fraca, mas ele continua sem entender. Então, é necessário que minha mãe me acompanhe para que ela me explique tudo depois. Não tenho vergonha dela, na verdade eu gosto quando ela vai comigo."

Entrevistado 4: "Vou ao médico junto com minha mãe e não tenho vergonha dela."

Entrevistado 6: "Costumo ir acompanhada com o meu marido e não tenho vergonha dele, pois ele me ajuda a entender tudo depois da consulta."

\section{CATEGORIA 5: SENTIMENTOS E EXPECTATIVAS FRENTE A UM MÉDICO FLUENTE EM LIBRAS}

Os entrevistados relataram como se sentiriam sendo atendidos por médicos fluentes em LIBRAS e nesse questionamento, demonstraram que ficariam felizes se fossem avaliados por médicos que utilizassem a Língua de Sinais para se comunicar.

Entrevistado 1: "Seria muito melhor, eu adoraria. É muito importante que os médicos saibam LIBRAS." 
Entrevistado 2: "Me sentiria muito feliz (face de alegria). Quando eu percebo que o médico não sabe LIBRAS, eu fico muito triste, com medo de morrer. Mas quando tem um intérprete ou o médico sabe, fico emocionada!"

Entrevistado 3: "Gostaria muito que o médico soubesse LIBRAS, seria ótimo."

Entrevistado 4: "É muito importante que o médico saiba LIBRAS, não somente por saber, mas para aprender a se comunicar com outro tipo de paciente, aquele que possui surdez."

Entrevistado 5: "É meio complicado, eu não conheço nenhum médico que saiba LIBRAS e até já me acostumei com isso. Acho que se o médico souber LIBRAS, é apenas um "pouquinho", mas mesmo assim é importante saber, eu ficaria muito feliz."

Entrevistado 7: "Eu me sentiria feliz se os médicos pudessem falar comigo, olhar para mim e explicar tudo o que preciso saber. Os médicos precisam parar de ficar somente oralizando e aprender pelo menos um pouco de LIBRAS."

\section{CATEGORIA 6: MEIOS DE COMUNICAÇÃO UTILIZADOS PELOS MÉDICOS DURANTE O ATENDIMENTO}

Os meios de comunicação mais prevalentes utilizados pelos médicos foram a verbalização e a escrita. Apenas um paciente surdo foi atendido por um médico que sabia LIBRAS, mas ainda assim, este preferia verbalizar e comunicar-se com a acompanhante do paciente, tornando inviável a comunicação eficaz e a formação de vínculo.

Entrevistado 1: "O médico usou apenas a escrita, no receituário. Não olhou para mim, apenas para o meu acompanhante."

Entrevistado 3: "O médico de Teresina, sabia um pouco de LIBRAS e a utilizou, mas falava mais com minha mãe."

Entrevistado 6: "O médico não usou LIBRAS e isso foi muito difícil. Ele escreveu, mas mesmo assim não entendi."

Entrevistado 7: "Quando vou ao médico ele apenas fala e escreve, utiliza o papel mostrando como tenho que usar o remédio, eu não entendo nada, mas já me acostumei com essa situação de desprezo."

Entrevistado 8: "O médico não sabe LIBRAS. Ele não escreveu, não desenhou, não olhou para mim nenhuma vez, apenas falava."
Foi observado que os entrevistados têm sentimentos de medo e frustração por não serem compreendidos por uma figura que na teoria deveria curá-los. Esses sentimentos surgem, pois não há comunicação efetiva entre o médico e o paciente surdo, prejudicando o atendimento e a criação de vínculo. Além disso, a ausência dessa comunicação efetiva, com o compartilhamento de informações adequado, não contribui com a resolução de problemas do paciente e dificulta a entrevista clínica, especialmente pelo déficit dos profissionais de saúde em LIBRAS $^{14}$.

O decreto 5.626/2005 que regulamentou a lei $n^{\circ} 10.436 / 2002$, estabelece no artigo 25 do capítulo VII, que o SUS deve garantir atenção integral à saúde, nos diversos níveis de complexidade e especialidades médicas, efetivando o atendimento às pessoas com surdez na rede de serviços por profissionais capacitados para o uso de LIBRAS ou para sua tradução e interpretação, bem como o apoio à capacitação e formação de profissionais do SUS para o uso da Língua de Sinais e sua tradução e interpretação ${ }^{(15)}$. Apesar disso, como os médicos relacionados à pesquisa não sabiam LIBRAS, foi observado que não houve explicações para os pacientes surdos durante a anamnese e/ou o exame físico. Isso faz com que os entrevistados busquem menos os serviços de saúde, tanto por essa falta de conhecimento em LIBRAS por parte dos médicos, quanto pela ausência de intérprete que pudesse intermediar a comunicação ${ }^{16}$.

O MCCP (Método Clínico Centrado na Pessoa) deve ser aplicado em toda consulta, sendo descrito como uma metodologia que visa alcançar a humanização da medicina ${ }^{17}$. Esse método, é importante dado que compreende um atendimento de maneira integral, considerando as necessidades, preocupações e vivências relacionadas à saúde dos pacientes, em que os usuários poderão relatar dados subjetivos como sentimentos, expectativas e conhecimentos a respeito da saúde/doença. Diante disso, uma das abordagens desse método é sobre o contexto familiar e o mesmo não foi abordado por nenhum dos médicos da pesquisa, sendo mais uma causa do distanciamento e da formação de sentimentos negativos dos pacientes surdos em relação aos médicos. Dessa forma, os pacientes procuram atendimento acompanhados de uma terceira pessoa, que apesar de culminar na ausência de privacidade, atua como mediador/facilitador do diálogo médico-paciente.

Como a comunicação é um instrumento de fundamental importância no acolhimento dos serviços de saúde, a escassez ou falha na mesma, impossibilita o trabalho dos profissionais, dificultando 0 atendimento inclusivo e humanizado ${ }^{18}$. Dessa forma, os pacientes surdos da pesquisa, demostraram que 
sentiriam grande satisfação, caso os médicos se comunicassem por meio da LIBRAS, uma vez que uma boa comunicação é relevante para a eficácia do exame clínico.

A falta de habilidade da utilização da Língua de Sinais associada com a falta de conhecimento de quem é a pessoa surda é um dos fatores que interferem na qualidade e assistência prestada pelos médicos. Ficou evidente nos depoimentos dos entrevistados 0 desconhecimento dos médicos sobre o uso da LIBRAS no momento da consulta, sendo utilizada a comunicação verbal na maior parte do atendimento. Outros entrevistados relataram que os médicos escreveram para explicar sobre o tratamento e conduta, porém os pacientes não entenderam, pois para estas pessoas o português é uma segunda língua, e como uma língua estrangeira, seu aprendizado é difícil ${ }^{19}$.

Portanto, é perceptível que a comunicação com os surdos é negligenciada nos sistemas de saúde, sendo indiscutível a necessidade de conhecimento da Língua de Sinais pelos médicos e outros profissionais da área para que haja avanços na assistência à saúde de forma integral e, consequentemente, ampliação da busca por atendimento ${ }^{20}$. É preciso que os profissionais da saúde reconheçam os pacientes com surdez e busquem habilidades comunicacionais adequadas a eles, pois esses fatores interferem na qualidade do atendimento ${ }^{21}$.

\section{CONCLUSÃO}

Ficou perceptível a partir deste trabalho, como os pacientes surdos se sentem diante do atendimento médico, tendo em vista a presença de uma barreira comunicacional que impede a formação de vínculo durante as consultas e, consequentemente, acolhimento holístico e inclusivo, interferindo de forma negativa no diagnóstico, tratamento e prognóstico dos mesmos.

Dessa forma, foi possível compreender os aspectos subjetivos de uma consulta na visão do paciente, bem como realizar uma autoanálise a respeito da importância do reconhecimento da LIBRAS para atender com qualidade as pessoas com surdez, servindo como facilitadores do acesso dos surdos aos serviços de saúde e garantindo que os princípios de universalidade, equidade e integralidade sejam garantidos.

Determinadas limitações foram perceptíveis no momento da entrevista, como o desconhecimento por parte de alguns surdos de um pequeno número de sinais da área da saúde, sendo necessário o entrevistador realizar datilologia para melhor compreensão dos mesmos. Um outro obstáculo, foi a chegada de alguns membros da família dos surdos no momento da entrevista, interferindo na conclusão de raciocínio do paciente durante as respostas.
Apesar disso, foi possível reconhecer o contexto social dos pacientes surdos entrevistados e as expectativas dos mesmos com relação ao processo comunicacional e garantia de seus direitos. Os pesquisadores, ao fazerem uso da LIBRAS durante as entrevistas, puderam de certa forma, reduzir a barreira comunicacional existente entre pessoas da área da saúde e pacientes surdos, sendo visualizado também a diminuição de sentimentos como tristeza e insegurança que estavam presentes nestes pacientes.

Sendo assim, sugere-se que novas pesquisas na área sejam conduzidas, a fim de reforçar a importância de LIBRAS na formação médica.

\section{Agradecimentos}

Aos participantes voluntários da pesquisa e familiares que nos acolheram em suas residências. Às pessoas de apoio afetivo dos surdos e conhecedoras da LIBRAS Íris Maria Oliveira da Silva e Clesivane do Socorro Silva do Nascimento, que nos auxiliaram na abordagem, interação e contato com as pessoas surdas.

\section{REFERÊNCIAS}

1. MUNARI, D. B. GODOY M. T. H. Recursos de relacionamento para profissionais de saúde: a boa comunicação com clientes e seus familiares em consultórios, ambulatórios e hospitais de Maria Tereza Maldonado e Paulo Canella. Revista Latino-Americana de Enfermagem, Ribeirão Preto, v. 12, n. 4, P. 689-690, jul./ago. 2004. Disponível em: Acesso em 12 mar. 2013.

2. SCHELLES, Suraia. A importância da linguagem não-verbal nas relações de liderança nas organizações, Revista Esfera, Brasília, n. 1 Jan./Jun. 2008, p.01-08.

3. GROSSEMAN S, PATRÍCIO ZM. A relação médico-paciente e o cuidado humano: subsídios para promoção da educação médica. Rev Bras Educ Méd. $2004 ; 28(2): 99-105$

4. BRASIL. (2002). Lei n 10.436, 24 de abril de 2002. Dispõe sobre a Língua Brasileira de Sinais - Libras e dá outras providências. Diário Oficial [da] República Federativa do Brasil, Brasília, DF, 24 abr 2002 Disponível em: <https://presrepublica.jusbrasil.com.br/legislacao/99492/lei-de-libras-lei10436-02>. Acesso em: 14 mar. 2018.

5. BRASIL. (2002) Lei. no 10.436, 2002. Disponível em: <http://periodicoscientificos.ufmt.br/ojs/index.php/revdia/article/viewFile/ 2851/1972 >. Acesso em: 12 março. 2018.

6. NORONHA, J.C.; PEREIRA, T.R. Princípios do sistema de saúde brasileiro. In: FUNDAÇÃO OSWALDO CRUZ. A saúde no Brasil em 2030: prospecção estratégica do sistema de saúde brasileiro: organização e gestão do sistema de saúde [online]. Rio de Janeiro: Fiocruz/Ipea/Ministério da Saúde/Secretaria de Assuntos Estratégicos da Presidência da República, 2013, vol. 3. p. 19-32. Disponivel em: <http://books.scielo.org/id/98kjw/pdf/noronha9788581100173-03.pdf>. Acesso em: 12 mar. 2018.

7. SAMPAIO, S.S.; RODRIGUES, F.W. Ética e Sigilo Profissional. Serv. Soc. Soc., São Paulo, n. 117, jan./mar. 201, p. 84-93.

8. BRASIL (2010). CARTILHA DO CENSO 2010. Pessoas com Deficiência. OLIVEIRA, Luiza Maria Borges. Secretaria de Direitos Humanos da Presidência da República (SDH/PR), Secretaria Nacional de Promoção dos Direitos da Pessoa com Deficiência (SNPD), Coordenação-Geral do Sistema de Informações sobre a Pessoa com Deficiência; Brasília: SDH PR/SNPD, 2012

9. CHAVEIRO, N.PORTO C. C.ALVES, B. M. Revisão de literatura sobre o atendimento ao paciente surdo pelos profissionais da saúde. Rev. Esc. Enferm. USP, São Paulo, set. 2008, pp. 578-583. Disponível em: http://www.scielo.br/pdf/reeusp/v42n3/v42n3a22.pdf. Acesso em: 14 maio 2018.

10. MACHADO, W.C.A. et al. The Teaching of Sign Language in Undergraduate Nursing Courses: A Descriptive Study as na Attribute to the Intergrality of Care. Health, v. 7, n. 14, p. 1824, 2015.

11. GIL, A. C. Métodos e técnicas de pesquisa social. São Paulo: Atlas, 1999. 
12. FONTANELLA, B.J. RICAS, J. \& TURATO, E.R. (2008). Saturation sampling in qualitative health research: theoretical contributions. Caderno de Saúde Pública, 24 (1), 17-27.

13. BARDIN, L. Análise de conteúdo. Lisboa: Edições 70, 2004.

14. ABREU, J. C.; FREITAS, J. M. R.; ROCHA, L. L. V. A percepção dos surdos em relação ao sistema de comunicação das unidades de atenção primária à saúde. Brazilian Journal of Surgery and Clinical Research, $v$. 9, n. 1, p. 06-11, 2015.

15. BRASIL. (2005). Decreto n. 5.626, de 22 de dezembro de 2005 regulamenta a Lei $n$. 10.436, sancionada em 24 de abril de 2002, a qual dispõe sobre a Língua Brasileira de Sinais Libras e o art. 18 da Lei $n$. 10.098, de 19 de dezembro de 2000. Diário Oficial da República Federativa do Brasil, Brasília, 23 dez. 2005. Disponível em: <http://www.planalto.gov.br/ccivil 03/ ato2004-

2006/2005/decreto/d5626. htm>. Acesso em: 14 mar. 2018.

16. STEINBERG, A.G; BARNETT, S.; MEADOR, H,E; WIGGINS, E.A ZAZOVE, P. Health care system accessibility. Experiences and perceptions of deaf people. J Gen Intern Med, v. 21, n. 3, Mar. 2006 p.260-266.

17. McWhinney IR. Clinical Method. In: McWhinney. A textbook of family medicine. New York:Oxford;1997. p.129-178.

18. OLIVEIRA, I. L.; PAULA, M.A. LIMA, F.; Para além das ideias e planos: processos e estratégias de comunicação. Propostas Conceituais para a Comunicação no Contexto Organizacional. São Caetano do Sul: Difusão Editora; Editora Senac Rio. P.67-77, 2012.

19. CHAVEIRO, Neuma; PORTO, Celmo Celeno; ALVES BARBOSA, Maria. Relação do paciente surdo com o médico. Brazilian Journal of Otorhinolaryngology, v. 75, n. 1, 2009.

20. CHAVEIRO, N.; BARBOSA, M. A. Assistência ao surdo na área de saúde como fator de inclusão social. Rev. Esc. Enferm., USP 2005, v. 39, p. 417-22.

21. McAleer M. Communicating effectively with deaf patients. Nurs Stand 2005; 20(19):51-4. 